\title{
A Flexible Phased-MIMO Array Antenna with Transmit Beamforming
}

\author{
Wen-Qin Wang ${ }^{1,2}$ and Huaizong Shao ${ }^{1}$ \\ ${ }^{1}$ The School of Communication and Information Engineering, University of Electronic Science and Technology of China (UESTC), \\ Chengdu 611731, China \\ ${ }^{2}$ The State Laboratory of Remote Sensing Science, Institute of Remote Sensing Applications, Chinese Academy of Sciences, \\ Beijing 100101, China \\ Correspondence should be addressed to Wen-Qin Wang, wqwang@uestc.edu.cn
}

Received 15 November 2011; Revised 23 January 2012; Accepted 24 January 2012

Academic Editor: Hon Tat Hui

Copyright (C) 2012 W.-Q. Wang and H. Shao. This is an open access article distributed under the Creative Commons Attribution License, which permits unrestricted use, distribution, and reproduction in any medium, provided the original work is properly cited.

\begin{abstract}
Although phased-array antennas have been widely employed in modern radars, the requirements of many emerging applications call for new more advanced array antennas. This paper proposes a flexible phased-array multiple-input multiple-output (MIMO) array antenna with transmit beamforming. This approach divides the transmit antenna array into multiple subarrays that are allowed to overlap each subarray coherently transmits a distinct waveform, which is orthogonal to the waveforms transmitted by other subarrays, at a distinct transmit frequency. That is, a small frequency increment is employed in each subarray. Each subarray forms a directional beam and all beams may be steered to different directions. The subarrays jointly offer flexible operating modes such as MIMO array which offers spatial diversity gain, phased-array which offers coherent directional gain and frequency diverse array which provides range-dependent beampattern. The system performance is examined by analyzing the transmitreceive beampatterns. The proposed approach is validated by extensive numerical simulation results.
\end{abstract}

\section{Introduction}

Phased-array antennas have been widely employed in different radars to provide electronic beam steering of radiated or received electromagnetic signals operating at the same frequency [1-3]. The beam can be steered to the desired direction by controlling the phase shifts across elements. It offers a directional gain which is useful for detecting/tracking weak targets and suppressing sidelobe interferences from other directions. If we want to focus the antenna beams in different directions, multiple antennas or a multi beam antenna are required [4]. The desire for new more advanced antenna array technologies has been driven by the requirements of many emerging applications [5-9].

A different strategy is to employ multiple-input multipleoutput (MIMO), which has received considerable attention in recent years, particularly in radar society [10-14]. The essence of the MIMO antenna array used in radars is to employ multiple antennas for emitting orthogonal waveforms or noncoherent waveforms and multiple antennas for receiving the echoes reflected by the target [15]. The MIMO array has two benefits: spatial diversity gain [16] and increased degree of freedoms (DOFs) due to the fact that a MIMO array realizes a virtual aperture that is larger than the physical array of its phased-array counterpart [17].

While MIMO array has many benefits over conventional phased-arrays, the former misses the directional gain. Intermediates between the two extremes were considered by jointly exploiting the benefits of the phased-array and MIMO array [18-21]. An idea by dividing the transmit array into multiple disjoint subapertures was introduced in [18]. Closeform expressions to achieve wide transmit beampattern were further developed [20]. Based on this work, a phasedMIMO technique was investigated in [21], which enjoys the advantages of the MIMO array without sacrificing the main advantages of the phased array in coherent processing gain. 
In this paper, we propose a flexible phased-MIMO antenna array with frequency diversity. We also divide the antenna array into multiple subarrays [21]. However, different from previous publications where an equal carrier frequency is employed in each subarray, here a distinct carrier frequency is employed in each subarray. That is, a small frequency increment is employed in each subarray. This provides an additional potential to suppress the grating lobes. This is particularly usefully when the subarrays are sparse (although randomly positioning the antennas can break up the grating lobes [22], it will bring higher sidelobes). More importantly, by optimally designing the frequency increments across the subarrays, we can obtain a range-dependent beampattern which is of great importance because it provides many new application potentials. The originality of this paper lies in the investigation of the tradeoff of spatial diversity gain, the spatial multiplexing gain, and the range-dependent effect of the phased-MIMO array.

In summary, the proposed new array antenna as compared to conventional phased-arrays has the following several unique features:

(1) enjoys the advantages of the phased-array providing a directional gain at the look direction;

(2) enjoys all the advantages of the MIMO arrays such as higher angular resolution [23], higher DOFs, and improved parameter identifiability [24];

(3) may provide range-dependent beampatterns which provides a potential to suppress range-dependent interferences;

(4) enables the use of beamforming on transmit;

(5) provides a potential to suppress the grating lobes;

(6) offers a tradeoff between angular resolution and robustness against interference and noise.

The remaining sections are organized as follows. Section 2 introduces the background and motivation of our work. In Section 3, we present the flexible phased-MIMO array and discuss several practical issues. Next, the transmit beamforming for the new array is derived in Section 4. Finally, numerical simulation examples are performed in Section 5 to evaluate the system performance. This paper is concluded in Section 6 with a short discussion of future work.

\section{Background and Motivation}

To facilitate the subsequent comparison between our approach and conventional phased array and MIMO array, this section makes an short overview of phased array and MIMO array.

2.1. Conventional Phased Array. Consider a phased array with $M$ transmit elements and $N$ receive elements, the transmitted signals can be represented by [25]

$$
\mathbf{a}^{*}\left(\theta_{0}\right) \cdot \phi(t),
$$

where $t$ is the range fast time, $\theta_{0}$ is the beam direction, $(\cdot)^{*}$ is a conjugate operator, and $\phi(t)$ is the baseband transmitted signal from each element. The transmit steering vector, and $\mathbf{a}(\theta)$ is expressed as

$$
\mathbf{a}(\theta)=\left[\begin{array}{llll}
1 & e^{-j\left(2 \pi f_{0} d \sin \theta / c_{0}\right)} & \cdots & e^{-j\left(2 \pi f_{0}(M-1) d \sin \theta / c_{0}\right)}
\end{array}\right]^{T},
$$

where $f_{0}, d, c_{0}$ and $(\cdot)^{T}$ denote the carrier frequency, element spacings, speed of light and matrix transpose, respectively. The signal seen at a far-field target with angle $\theta_{i}$ can be given by

$$
\mathbf{a}^{T}\left(\theta_{i}\right) \cdot \mathbf{a}^{*}\left(\theta_{0}\right) \cdot \phi(t),
$$

when $\theta_{i}=\theta_{0}$, we can obtain a directional gain of $M$ at the look angle of $\theta_{0}$.

Suppose there are multiple interferences $\left(\theta_{i}\right)$ near the desired target $\theta_{0}$. The received signals can then be expressed as

$$
\mathbf{x}_{\mathbf{p a}}(t)=\alpha_{0} M \mathbf{b}\left(\theta_{0}\right) \phi(t)+\sum_{i} \alpha_{i} \mathbf{a}^{T}\left(\theta_{i}\right) \mathbf{a}^{*}\left(\theta_{0}\right) \mathbf{b}\left(\theta_{i}\right) \phi(t)+\mathbf{n}(t),
$$

where $\alpha_{i}$ denotes the complex amplitude of the $i$ th source, $\mathbf{b}(\theta)$ is an $N \times 1$ propagation vector due to the propagation delays from a source to the receive elements, and $\mathbf{n}(l)$ is the $N \times 1$ additive white Gaussian noise vector with zero mean and covariance matrix $\sigma_{n}^{2} \mathbf{I}$ with $\mathbf{I}$ being an identity matrix. The $\mathbf{b}(\theta)$ similarly defined as the transmit steering vector, and $\mathbf{a}(\theta)$ is the receive steering vector. By matched-filtering the received signal to the transmitted signal $\phi(t)$, we can get

$$
\mathbf{y}_{\mathrm{pa}} \triangleq \alpha_{0} \mathbf{u}\left(\theta_{0}\right)+\sum_{i} \alpha_{i} \mathbf{u}\left(\theta_{i}\right)+\mathbf{n},
$$

where the subscript "pa" denotes the general phased array and the virtual steering vector is

$$
\mathbf{u}_{\mathrm{pa}}(\theta) \triangleq \beta \cdot \mathbf{b}(\theta)
$$

with $\beta$ being the transmit beampattern directional gain. It can be easily proved that $\mathbf{n}$ has zero mean and covariance matrix $\sigma_{n}^{2} \mathbf{I}$.

2.2. General MIMO Array. Consider a colocated MIMO array with $M$ transmit antennas and $N$ receive antennas, where each transmit antenna sends out a different waveform $s_{m}(t), m=1,2, \ldots, M$. Suppose there is a target located at the look direction $\theta_{0}$ along with $D$ interference sources locating at $\theta_{i}, i=1,2, \ldots, D$. The baseband signals at the $N$-element receive array can be written as

$$
\mathbf{x}_{\text {mimo }}(t)=\alpha_{0} \mathbf{b}\left(\theta_{0}\right) \mathbf{a}^{\mathrm{T}}\left(\theta_{0}\right) \mathbf{s}(t)+\sum_{i=1}^{D} \alpha_{i} \mathbf{b}\left(\theta_{i}\right) \mathbf{a}^{\mathbf{T}}\left(\theta_{i}\right) \mathbf{s}(t)+\mathbf{n}(t),
$$

where $\mathbf{a}(\theta)$ and $\mathbf{b}(\theta)$ are the transmit and receive steering vectors associated with the direction $\theta, \mathbf{s}(t)$ is the $M \times 1$ vector collecting all transmitted waveforms, and the subscripts "mimo" denotes the general MIMO arrays. 
Since the signals transmitted from each element are orthogonal, we have

$$
\int_{T_{P}} \mathbf{s}(t) \mathbf{s}^{H}(t) \mathrm{d} t=\mathbf{I}_{M \times M},
$$

where $T_{p}$ is the pulse duration, $(\bullet)$ is a complex matrix conjugate transpose, and $\mathbf{I}_{M \times M}$ is an $M \times M$ identity matrix. The received signals can be processed by a matched filter, which outputs an $M N \times 1$ vector given by

$$
\begin{aligned}
\mathbf{y}_{\text {mimo }} & =\left[\int_{T_{p}} \mathbf{x}_{\text {mimo }}(t) \mathbf{s}^{H}(t) \mathrm{d} t\right]\left[\int_{T_{0}} \mathbf{s}(t) \mathbf{s}^{H}(t) d t\right]^{-1} \\
& =\alpha_{0} \mathbf{a}\left(\theta_{0}\right) \otimes \mathbf{b}\left(\theta_{0}\right)+\sum_{i=1}^{D} \alpha_{i} \mathbf{a}\left(\theta_{i}\right) \otimes \mathbf{b}\left(\theta_{i}\right)+\mathbf{n},
\end{aligned}
$$

where $\otimes$ denotes the Kronker product. The joint transmitreceive steering vector can then be expressed as

$$
\mathbf{u}_{\text {mimo }}(\theta) \doteq \mathbf{a}(\theta) \otimes \mathbf{b}(\theta) .
$$

Comparing (6) and (10) we can see that MIMO array provides increased DOFs, higher angular resolution and better detection performance [26]. However, since orthogonal waveforms are employed in the transmitter [27], transmit beamforming cannot be employed in MIMO arrays [28]; therefore, it lacks robustness again noise and interference. It is thus necessary to jointly exploit the advantages of phased array and MIMO array, along with other techniques. This is just the motivation of this paper.

\section{New Phased-MIMO Array}

In this section, we introduce the new phased-MIMO array and discuss several practical implementation issues.

3.1. Flexible Phased-MIMO Array. Like the general phasedMIMO array discussed in the literature [18-21], we divide the transmit array into multiple $(K)$ subarrays which can be disjoint or overlapped, as shown in Figure 1. Each transmit subarray can be composed of any number of elements ranging from 1 to $M$. However, unlike the general phasedMIMO array discussed in the literature, in this paper all elements in each subarray are used to coherently emit the signal $s_{k}(t)$ at a distinct carrier frequency. That is, a small frequency increment is employed between the subarrays. A beam can be formed by each subarray towards a certain direction. The beamforming weight vector can be properly designed to maximize the coherent processing gain. At the same time, different waveforms are transmitted by different subarrays.

Suppose the $k$ th subarray consists of $N_{k}<M$ transmit elements, the equivalent baseband signal model can be modeled as

$$
\sqrt{\frac{M}{K}} \phi_{k}(t) \tilde{w}_{k}^{*}, \quad k=1,2, \ldots, K
$$

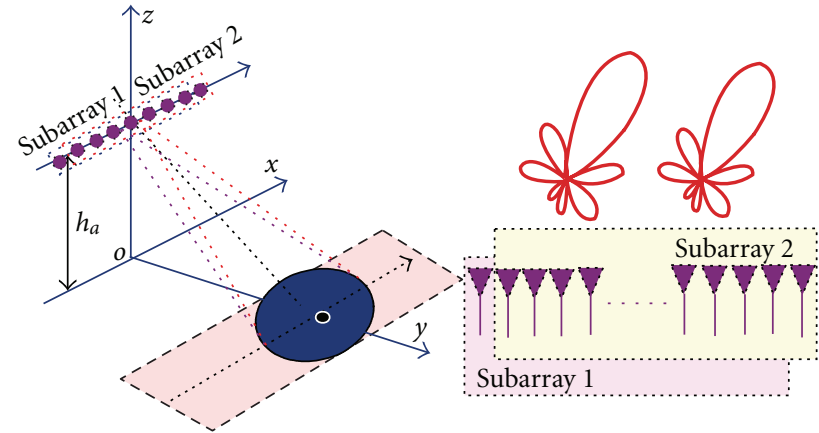

FIGURE 1: Illustration of the flexible phased-MIMO antenna array.

with

$$
\int_{T_{p}} \phi_{k}(t) \phi_{k^{\prime}}^{H}(t) \mathrm{d} t=\delta\left(k-k^{\prime}\right),
$$

where $K$ is the number of subarrays, $\widetilde{w}_{k}$ is the $M \times 1$ unitnorm complex vector which consists of $N_{k}$ beamforming weights corresponding to the active antennas of the $k$ th subarray, that is, the number of nonzeros in $\widetilde{w}_{k}$ equals to $N_{k}$ and the number of zeros equals to $M-N_{k}$. Note that $\sqrt{M / K}$ is used to obtain an identical transmission power constraint for subsequent comparison, which means the transmit energy within one pulse repetition interval (PRI) is given by

$$
\int_{T_{p}} s_{k}^{H}(t) s_{k}(t) \mathrm{d} t=\frac{M}{K} .
$$

Suppose the carrier frequency in each subarray is

$$
f_{k}=f_{0}+(k-1) \Delta f,
$$

where $\Delta f$ is the frequency offset. The signal reflected by a target located at direction $\theta$ can be modeled as [21]

$$
s_{r}(t, \theta)=\sqrt{\frac{M}{K}} \sigma(\theta) \sum_{k=1}^{K} \mathbf{w}_{k}^{H} \mathbf{a}_{k}(\theta) e^{-j 2 \pi f_{k} \tau_{k}(\theta)} \phi_{k}(t),
$$

where $\sigma(\theta)$ is the target reflection coefficient, the $\mathbf{w}_{k}$ and $\mathbf{a}_{k}(\theta)$ which contain only the elements corresponding to the active elements of the $k$ th subarray are the $N_{k} \times 1$ beamforming vector and steering vector, and the slant range $(r)$ dependent $\tau_{k}(\theta)$ is the required signal propagation time for the $k$ th subarray. After matched-filtering the received signal by each of the waveform $\phi_{k}(t)$, we can get an $K N \times 1$ data vector

$$
s_{r}(t, \theta)=\sqrt{\frac{M}{K}} \sigma(\theta)[\mathbf{c}(\theta) \odot \mathbf{d}(\theta, r)]^{T} \otimes \mathbf{b}(\theta),
$$

where $\odot$ is the Hadamard (element-wise) product, $\otimes$ is the Kronker product. The $\mathbf{c}(\theta)$ and $\mathbf{d}(\theta)$ are $K \times 1$ vectors defined as

$$
\begin{aligned}
\mathbf{c}(\theta) & \doteq\left[\mathbf{w}_{1}^{H} \mathbf{a}_{\Theta_{1}}(\theta), \mathbf{w}_{2}^{H} \mathbf{a}_{\Theta_{2}}(\theta), \ldots, \mathbf{w}_{K}^{H} \mathbf{a}_{\Theta_{K}}(\theta)\right]^{T}, \\
\mathbf{d}(\theta, r) & \doteq\left[e^{-j 2 \pi f_{1} \tau_{1}(\theta)}, e^{-j 2 \pi f_{2} \tau_{2}(\theta)}, \ldots, e^{-j 2 \pi f_{K} \tau_{K}(\theta)}\right]^{T}, \\
\phi_{K}(t) & \doteq\left[\phi_{1}(t), \phi_{2}(t), \ldots, \phi_{K}(t)\right]^{T} .
\end{aligned}
$$


Suppose that a target is located at angle $\theta_{0}$ and $D$ interference sources at $\theta_{i}$. By matched-filtering the received signal corresponding to each of the waveform $\phi_{k}(t)$, we can get an $K N \times 1$ data vector

$$
\mathbf{y}=\sqrt{\frac{M}{K}} \alpha\left(\theta_{0}, r_{0}\right) \mathbf{u}\left(\theta_{0}\right)+\sum_{i=1}^{D} \sqrt{\frac{M}{K}} \alpha_{i} \mathbf{u}\left(\theta_{i}, r_{i}\right)+\tilde{\mathbf{n}}
$$

with virtual steering vector

$$
\mathbf{u}_{\text {pa-mimo }}(\theta, r)=[(\mathbf{c}(\theta) \odot \mathbf{d}(\theta, r))] \otimes \mathbf{b}(\theta),
$$

where $\tilde{\mathbf{n}}$ is the $K N \times 1$ noise term. Specially, if $\Delta f=0$ is chosen for the progressively frequency increment between subarrays, (19) simplies to the general phased-MIMO array.

3.2. Discussions. In the following, we discuss several practical issues related to the flexible array antenna.

3.2.1. Flexible Performance Specifics. First, the flexible array is a tunable system that offers a continuum of operating modes from the phased array, MIMO array and frequency diverse array.

(1) If $\Delta f=0$ and $K=1$ are chosen for the array, it is a general phased array which offers a coherent directional gain.

(2) If $\Delta f=0$ and $K=M$ are chosen for the array and orthogonal waveforms are employed in each subarray, it is a general MIMO array which offers a spatial diversity gain.

(3) If $\Delta f=0$ and $1<K<M$ are chosen for the array and orthogonal waveforms are employed in each subarray, it is a general phased-MIMO array which is a hybrid array that offers some of the directional gain of the phased-array and also some of the interference rejection capability of the MIMO array [20].

(4) If $\Delta f \neq 0$ and $K=M$ are chosen for the array and orthogonal waveforms are employed in each subarray, it is a MIMO array with frequency diversity. It has been proved in [29-32] that the use of frequency diversity in MIMO array is useful.

(5) If $\Delta f \neq 0$ and $K=M$ are chosen for the array and the same waveform is employed in each subarray, it is a frequency diverse array which provides a rangedependent transmit beampattern [33-36].

If $\Delta f \neq 0$ and $1<K<M$ are chosen for the array, it can be formed to a phased-MIMO array with frequency diversity when orthogonal waveforms are employed in each subarray or the signals transmitted by each subarray are orthogonal in frequency. This can be used to suppress range-dependent interferences [37].

Suppose the number of transmit elements are equal to the number of receive element, that is, $M=N$. Considering the DOF (Note that the DOF defined in MIMO radar may be different from that defined in MIMO communication. In most of MIMO radar literature, the DOF is defined as the number of independent spatial samples. Therefore, for an $M \times N$ MIMO radar system, there will be $M \times N$ DOFs available while no more than $\min (M, N)$ DOFs are available for an $M \times N$ MIMO communication system) performance, we have the following.

(1) If $\Delta f=0$ and $K=1$ are chosen for the array, it has $N$ DOFs because it trades off $N-1$ DOFs to attain a directional gain of $N$.

(2) If $\Delta f=0$ and $K=M$ are chosen for the array and orthogonal waveforms are employed in each subarray, it has $2 N-2$ DOFs without directional gain.

(3) If $\Delta f=0$ and $1<K<M$ are chosen for the array and orthogonal waveforms are employed in each subarray, it trades off $N-K$ DOFs to attain a directional gain of $N-K+1$. Then there is $(2 N-$ 1) $-(N-K)=N+K-1$ DOFs.

(4) If $\Delta f \neq 0$ and $K=M$ are chosen for the array and orthogonal waveforms are employed in each subarray, it has $2 N-2$ DOFs without directional gain.

(5) If $\Delta f \neq 0$ and $K=M$ are chosen for the array and the same waveform is employed in each subarray, when the signals transmitted by each subarray are not orthogonal in frequency it has $N$ DOFs because it trades off $N-1$ DOFs to attain a directional gain of $N$; however, when the signals transmitted by each subarray are orthogonal in frequency it has $2 \mathrm{~N}-2$ DOFs without directional gain.

(6) If $\Delta f \neq 0$ and $1<K<M$ are chosen for the array and orthogonal waveforms are employed in each subarray, it has $N+K-1$ DOFs with a directional gain of $N-K+1$.

3.2.2. Design Specifics. One design specific is the grating lobe suppression which can be achieved by coherently combining the $N_{k}$ subbeams formed at different frequencies. Suppose the target's angle is $\theta_{0}$, the receive beam at the $k$ th frequency can be represented by

$$
F_{k}(\theta)=\sum_{n=1}^{N_{k}} \exp \left[-j \frac{2 \pi}{c_{0}} f_{k} L(n-1)\left(\sin \theta-\sin \theta_{0}\right)\right] \text {, }
$$

where $L$ is the spacings between two subarrays. The sidelobe decorrelation performance can be evaluated by the cross correlation function of two beams formed at two different frequencies [38-40]

$$
\begin{aligned}
C_{k, k+1}(\theta) & =E\left\{F_{k}(\theta) F_{k+1}^{*}(\theta)\right\} \\
& \cong N_{k} \frac{\sin \left[\Delta f\left(\sin \theta-\sin \theta_{0}\right) / 2\right]}{\Delta f\left(\sin \theta-\sin \theta_{0}\right) / 2} .
\end{aligned}
$$

Thus, the two beams are decorrelated in the sidelobe region when

$$
\Delta f=\frac{c_{0}}{(N-1) L\left(\sin \theta-\sin \theta_{0}\right)} .
$$


It is concluded in [38] that, the average sidelobe level is not impacted by the $\Delta f$ variations and the peak sidelobe level can be reduced by increasing the $\Delta f$.

On the other hand, to avoid target decorrelation happen in each subarray, the $\Delta f$ is limited by [41]

$$
\Delta f<\frac{c_{0}}{2 \zeta \sin (\theta)}
$$

where $\zeta$ is the length of the target, and $2 \zeta \sin (\theta)$ is the length of the target projected along the radar boresight. Note that this is a highly simplified model which is independent on the transmitted frequency. Moreover, to avoid signal decorrelation happen in each subarray, the $\Delta f$ is also limited by [42]

$$
\Delta f<\frac{1}{T_{p}} .
$$

Otherwise, the signals transmitted by each subarray will be orthogonal in frequency. Certainly, if frequency orthogonality between the subarrays is desired, the $\Delta f$ should be

$$
\begin{aligned}
\frac{1}{T_{p}} \int_{-T_{p} / 2}^{T_{p} / 2} \exp \left(j 2 \pi f_{k} t\right) \exp \left(j 2 \pi f_{k^{\prime}} t\right) \mathrm{d} t \\
\quad=\operatorname{sinc}\left[\pi\left(k-k^{\prime}\right) \Delta f T_{p}\right] \\
\quad=0
\end{aligned}
$$

That is

$$
\Delta f=\frac{i}{T_{p}}, \quad i=1,2,3, \ldots
$$

\section{Transmit-Receive Beamforming}

To simplify the discussion on the transmit-receive beampattern performance, we rewrite the output of the matched filter for the several arrays introduced previously as

$$
\begin{aligned}
\mathbf{y} & =\alpha_{0} \mathbf{u}\left(\theta_{0}, r_{0}\right)+\sum_{i=1}^{D} \alpha_{i} \mathbf{u}\left(\theta_{i}, r_{i}\right)+\mathbf{n} \\
& =\alpha_{0} \mathbf{u}\left(\theta_{0}, r_{0}\right)+\mathbf{d}
\end{aligned}
$$

where $\mathbf{u}(\theta, r)$ can refer to any of (6), (10) and (19), and $\mathbf{d}$ denotes the interference plus noise signal.

Transmit beamforming can be employed for different subarrays such that certain beampattern and/or transmit power requirements are satisfied. In this paper, we use the conventional nonadaptive transmit-receive beamforming technique which is widely used in antenna array systems because it is known to be optimal in the sense that it provides the highest possible output signal-to-noise ratio (SNR) in the background of white Gaussian noise [43]. When the conventional nonadaptive beamformer is employed, the transmit beamformer vector can be written as

$$
\mathbf{w}_{k}=\frac{\mathbf{a}_{k}\left(\theta_{0}\right)}{\left\|\mathbf{a}_{k}\left(\theta_{0}\right)\right\|}, \quad k=1,2,3, \ldots, K .
$$

Similarly, the receive beamformer weight vector is

$$
\mathbf{w}_{R} \triangleq \mathbf{u}\left(\theta^{0}, r^{0}\right)
$$

The corresponding normalized transmit-receive beampattern is expressed in

$$
\begin{aligned}
G(\theta) & \doteq \frac{\left|\mathbf{w}_{R}^{H} \mathbf{u}(\theta, r)\right|^{2}}{\left|\mathbf{w}_{R}^{H} \mathbf{u}\left(\theta_{0}, r_{0}\right)\right|^{2}} \\
& =\frac{\left|\mathbf{u}^{H}\left(\theta_{0}, r_{0}\right) \mathbf{u}(\theta, r)\right|^{2}}{\left\|\mathbf{u}\left(\theta_{0}, r_{0}\right)\right\|^{2}} \\
& =\frac{\left|\mathbf{a}_{K}^{H}\left(\theta_{0}\right) \mathbf{a}_{K}(\theta)\right|^{2}\left|\mathbf{d}^{H}\left(\theta_{0}, r_{0}\right) \mathbf{d}(\theta, r)\right|^{2}\left|\mathbf{b}^{H}\left(\theta_{0}\right) \mathbf{b}(\theta)\right|^{2}}{\left\|\mathbf{a}_{K}\left(\theta_{0}\right)\right\|^{4}\left\|\mathbf{d}\left(\theta_{0}, r_{0}\right)\right\|^{4}\left\|\mathbf{b}\left(\theta_{0}\right)\right\|^{4}}
\end{aligned}
$$

where a uniform linear array (ULA) is assumed. [21].

Inspecting (30) we can obtain the following conclusions

(1) If $\Delta f=0$ and $K=1$ are chosen for the array, the transmit-receive beampattern simplifies to

$$
G_{\mathrm{pa}}(\theta) \triangleq \frac{\left|\mathbf{a}^{H}(\theta) \mathbf{a}(\theta)\right|^{2}}{M^{2}} \cdot \frac{\left|\mathbf{b}^{H}(\theta) \mathbf{b}(\theta)\right|^{2}}{N^{2}} .
$$

(2) If $\Delta f=0$ and $K=M$ are chosen for the array and orthogonal waveforms are employed in each subarray, the transmit-receive beampattern simplifies to

$$
G_{\text {mimo }}(\theta) \triangleq \frac{\left|\mathbf{a}^{H}(\theta) \mathbf{a}(\theta)\right|^{2}}{M^{2}} \cdot \frac{\left|\mathbf{b}^{H}(\theta) \mathbf{b}(\theta)\right|^{2}}{N^{2}}
$$

(3) If $\Delta f=0$ and $1<K<M$ are chosen for the array and orthogonal waveforms are employed in each subarray, the transmit-receive beampattern simplifies to

$$
\begin{aligned}
& G_{\text {pa-mimo }}(\theta, r) \\
& \quad \triangleq \frac{\left|\mathbf{a}_{K}^{H}(\theta) \mathbf{a}_{K}(\theta)\right|^{2}}{M^{2}} \cdot \frac{\left|\mathbf{d}^{H}(\theta, r) \mathbf{d}(\theta, r)\right|^{2}}{K^{2}} \cdot \frac{\left|\mathbf{b}^{H}(\theta) \mathbf{b}(\theta)\right|^{2}}{N^{2}} .
\end{aligned}
$$

In this case, the $\mathbf{d}(\theta)$ is range independent.

(4) If $\Delta f \neq 0$ and $K=M$ are chosen for the array and orthogonal waveforms are employed in each subarray, the transmit-receive beampattern simplifies to

$$
\begin{aligned}
& G_{\text {fd-mimo }}(\theta, r) \\
& \quad \triangleq \frac{\left|\mathbf{a}^{H}(\theta) \mathbf{a}(\theta)\right|^{2}}{M^{2}} \cdot \frac{\left|\mathbf{d}^{H}(\theta, r) \mathbf{d}(\theta, r)\right|^{2}}{K^{2}} \cdot \frac{\left|\mathbf{b}^{H}(\theta) \mathbf{b}(\theta)\right|^{2}}{N^{2}} .
\end{aligned}
$$




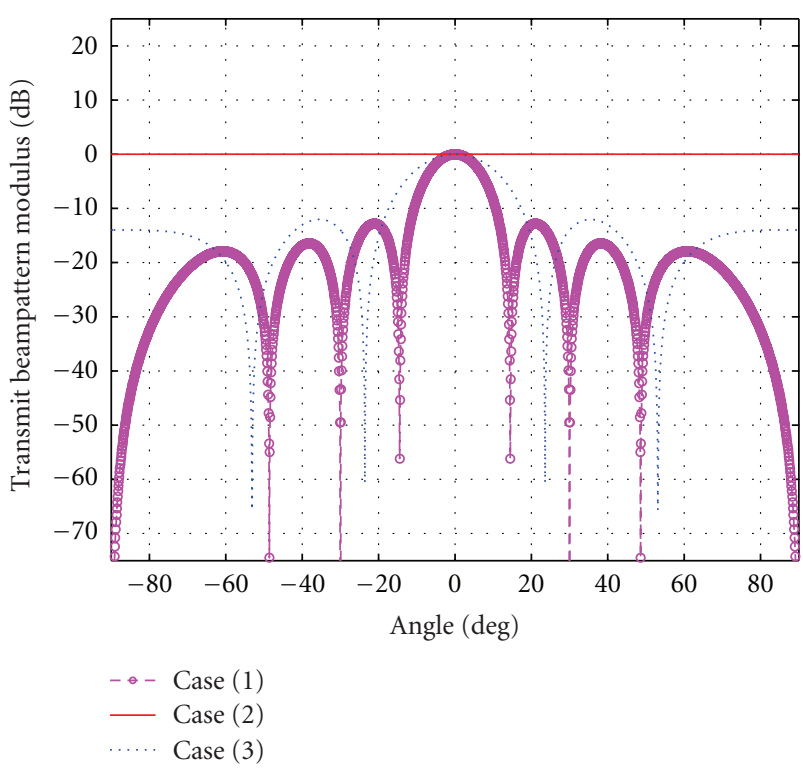

Figure 2: The comparative transmit beampatterns when $\Delta f=0$.

(5) If $\Delta f \neq 0$ and $K=M$ are chosen for the array and the same waveform is employed in each subarray, the transmit-receive beampattern simplifies to

$$
\begin{aligned}
& G_{\mathrm{fs}}(\theta, r) \\
& \quad \triangleq \frac{\left|\mathbf{a}^{H}(\theta) \mathbf{a}(\theta)\right|^{2}}{M^{2}} \cdot \frac{\left|\mathbf{d}^{H}(\theta, r) \mathbf{d}(\theta, r)\right|^{2}}{K^{2}} \cdot \frac{\left|\mathbf{b}^{H}(\theta) \mathbf{b}(\theta)\right|^{2}}{N^{2}} .
\end{aligned}
$$

(6) If $\Delta f \neq 0$ and $1<K<M$ are chosen for the array and orthogonal waveforms are employed in each subarray, the transmit-receive beampattern simplifies to

$$
\begin{aligned}
& G_{\text {fp-mimo }}(\theta, r) \\
& \qquad \triangleq \frac{\left|\mathbf{a}_{K}^{H}(\theta) \mathbf{a}_{K}(\theta)\right|^{2}}{(M-K+1)^{2}} \cdot \frac{\left|\mathbf{d}^{H}(\theta, r) \mathbf{d}(\theta, r)\right|^{2}}{K^{2}} \cdot \frac{\left|\mathbf{b}^{H}(\theta) \mathbf{b}(\theta)\right|^{2}}{N^{2}} .
\end{aligned}
$$

It can be noticed that, when $\Delta f \neq 0$, the transmitreceive beampattern will be range dependent. Although (31) and (32), (34) and (35) are equivalent to each other, but they have different transmit beampattern and diversity beampattern. Therefore, the new phased-MIMO array as compared to the general phased-MIMO array provides more flexible operation modes such as phased-array, MIMO array, frequency diverse array, phased-MIMO array, and frequency diverse phased-MIMO array. Different transmit/receive or transmit-receive beampatterns can be obtained by applying different beamformer weight vectors.

\section{Simulation Results and Discussions}

First, we suppose $\Delta f=0$ which means that the array is just the general phased-MIMO array. Suppose the following

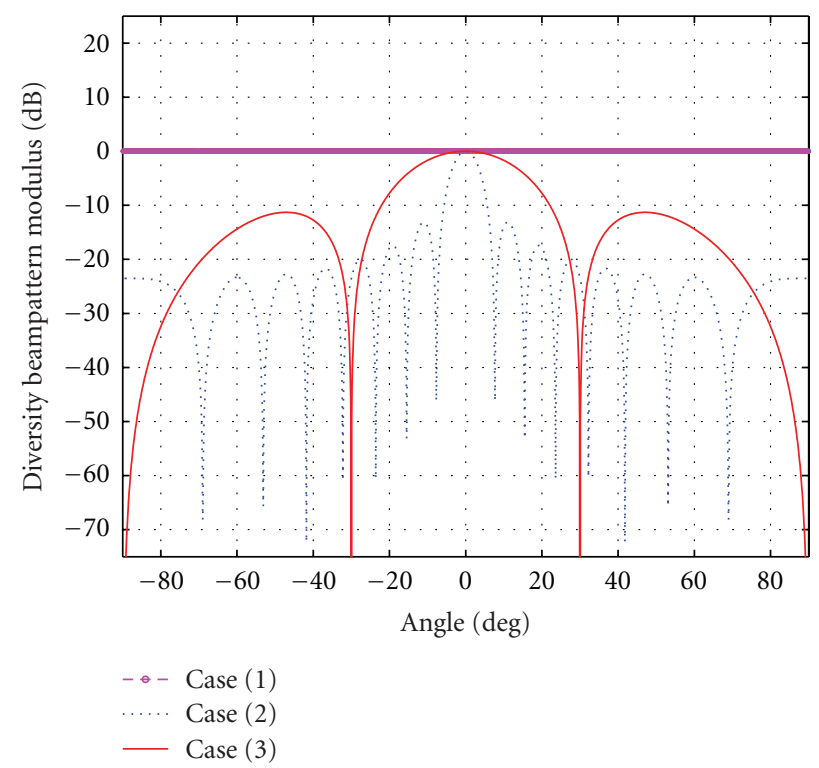

FIGURE 3: The comparative diversity beampatterns when $\Delta f=0$.

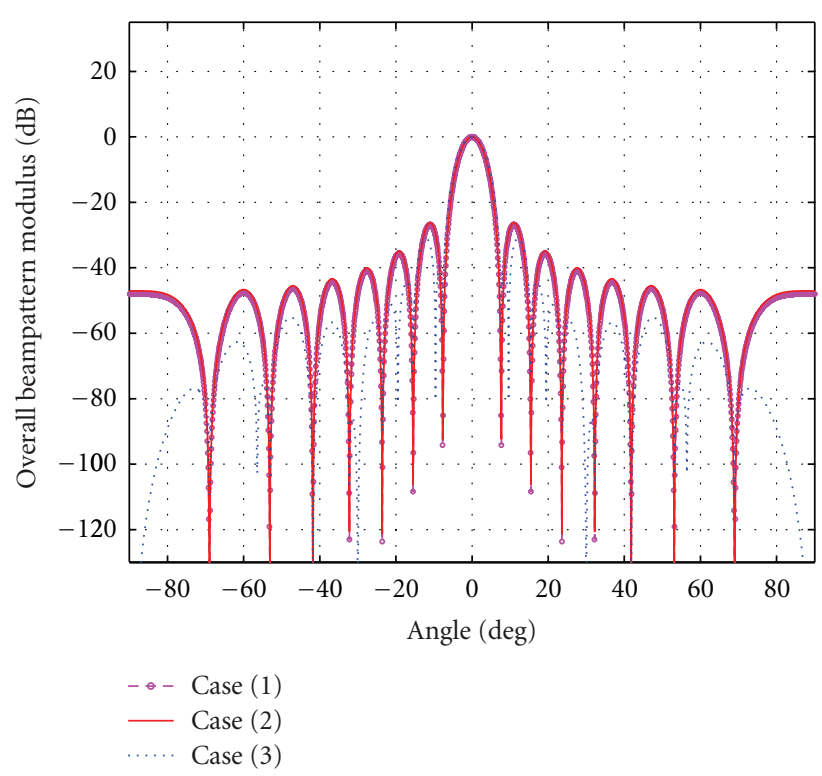

FIGURE 4: The comparative overall transmit-receive beampatterns when $\Delta f=0$.

simulation parameters: $M=N=15, f_{0}=10 \mathrm{GHz}$, $d=\lambda / 2$ and $K=4$. In this case, the beampatterns are range independent. Figure 2 shows the comparative transmit beampatterns. It can be noticed that the case (1) (phasedarray) has a directional gain but the case (2) (MIMO array) has no directional gain. Figure 3 shows the comparative diversity beampatterns. It can be noticed that the case 1 has no diversity gain but the case (2) has a diversity gain. Figure 4 shows the comparative overall transmit-receive beampatterns. It can noticed that the cases (1) and (2) have almost the same overall transmit-receive beampattern; 


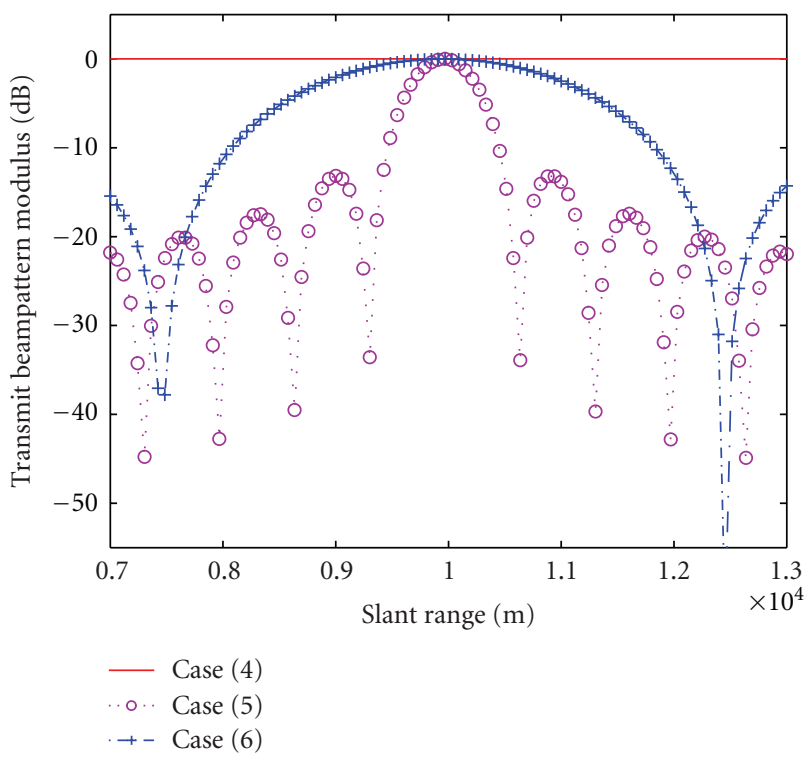

FIGURE 5: The comparative transmit beampatterns in range dimension when $\Delta f \neq 0$.

however, the case (3) (phased-MIMO array) has a better overall transmit-receive beampattern.

Next, we apply frequency diversity to the array, that is, $\Delta f \neq 0$. In this case, the beampatterns will be range dependent. It can be easily proved that the beampatterns in the angle dimension are still the same to the beampatterns when $\Delta f=0$. So, here we consider only the beampatterns in the range dimension. Suppose $\Delta f=30 \mathrm{kHz}$ and the beam direction range is $r_{0}=10 \mathrm{~km}$. Figure 5 shows the comparative transmit beampatterns in range dimension. It can be noticed that the case (4) (MIMO array with frequency diversity) provides no directional gain in range dimension but the case (5) (frequency diverse array) provides a directional gain. The case (6) (phased-MIMO array with frequency diversity) also provides a directional gain in range dimension, but it has a wider mainlobe due to its smaller aperture length. Figure 6 gives the comparative overall beampatterns in range dimension. It can be noticed that cases (4) and (5) have exactly the same overall transmitreceive beampattern in range dimension. At the same time, the case (6) has a wider mainlobe because fewer $(K=4)$ diverse frequencies are employed in it. Therefore, considering also the system complexity, $K=3$ or 4 may be the most appropriate value for the $K$.

From the above simulation results we can conclude that, the use of a frequency increment $\Delta f$ in each subarray can provide a range-dependent beampattern. Taking case (5) as an example, the beampattern at a far field observation with angle $\theta$ and range $r$ can be represented by

$$
\begin{aligned}
y(\theta, r) & =\sum_{m=1}^{M} e^{j\left(-2 \pi f_{0}(m-1) L \sin \theta / c_{0}-2 \pi(m-1)^{2} \Delta f L \sin \theta / c_{0}+2 \pi r(m-1) \Delta f / c_{0}\right)} \\
& \approx \sum_{m=0}^{M-1} e^{j 2 m\left(-\pi f_{0} L \sin \theta / c_{0}+\pi r \Delta f / c_{0}\right)}=\frac{e^{j M\left(-\pi f_{0} L \sin \theta / c_{0}+\pi r \Delta f / c_{0}\right)} \sin \left[M\left(-\left(\pi f_{0} L \sin \theta / c_{0}\right)+\left(\pi r \Delta f / c_{0}\right)\right)\right]}{e^{j\left(-\pi f_{0} L \sin \theta / c_{0}+\pi r \Delta f / c_{0}\right) \sin \left[\left(-\left(\pi f_{0} L \sin \theta / c_{0}\right)+\left(\pi r \Delta f / c_{0}\right)\right)\right]}},
\end{aligned}
$$

where the element factor is omitted since only the array pattern is the subject of our analysis. The normalized beamformer response is

$$
\begin{aligned}
& |y(\theta, r)| \\
& \quad=\frac{1}{M}\left|\frac{\sin \left[M\left(-\pi f_{0} L \cos \theta / c_{0}+\pi r \Delta f / c_{0}\right)\right]}{\sin \left(-\pi f_{0} L \cos \theta / c_{0}+\pi r \Delta f / c_{0}\right)}\right| .
\end{aligned}
$$

Therefore, the range-dependent beampattern can provide local maxima at different ranges.

Suppose the following parameters: $M=N=15, f_{0}=$ $10 \mathrm{GHz}, \Delta f=30 \mathrm{kHz}$ and $L=0.5 \lambda$, Figure 7 shows the corresponding transmit beampattern when compared to the conventional phased-array. It can be noticed that the use of frequency increment in each subarray offers a range-dependent beampattern. We further investigated the case when the subarrays are displaced more than a half wavelength apart from each other. As an example, $L=$ $1.5 \lambda$ is chosen. The corresponding comparative transmit beampattern is shown in Figure 8. Each beampattern is repeated three times within the spatial domain due to spatial aliasing. This is because the interelement spacing is three times half a wavelength. Range-dependent beampattern can also be noticed from the frequency diverse array. This range-dependent beampattern may provide many potential advantages. Typical applications include suppressing rangedependent clutter [37] and improving range resolution for synthetic aperture radar (SAR) $[44,45]$.

\section{Conclusion}

This paper proposes a flexible phased-array MIMO array antenna with beamforming on transmit, which applies frequency diversity to the phased-MIMO array. This approach divides the transmit antenna array into multiple subarrays that are allowed to overlap, each subarray coherently transmits a distinct waveform, which is orthogonal to the waveforms transmitted by other subarrays, at a distinct transmit frequency. That is, a small frequency increment is employed in each subarray. Each subarray forms a directional beam and all beams may be steered to different directions. The subarrays jointly offer flexible operating modes such as MIMO array which offers spatial diversity gain and 


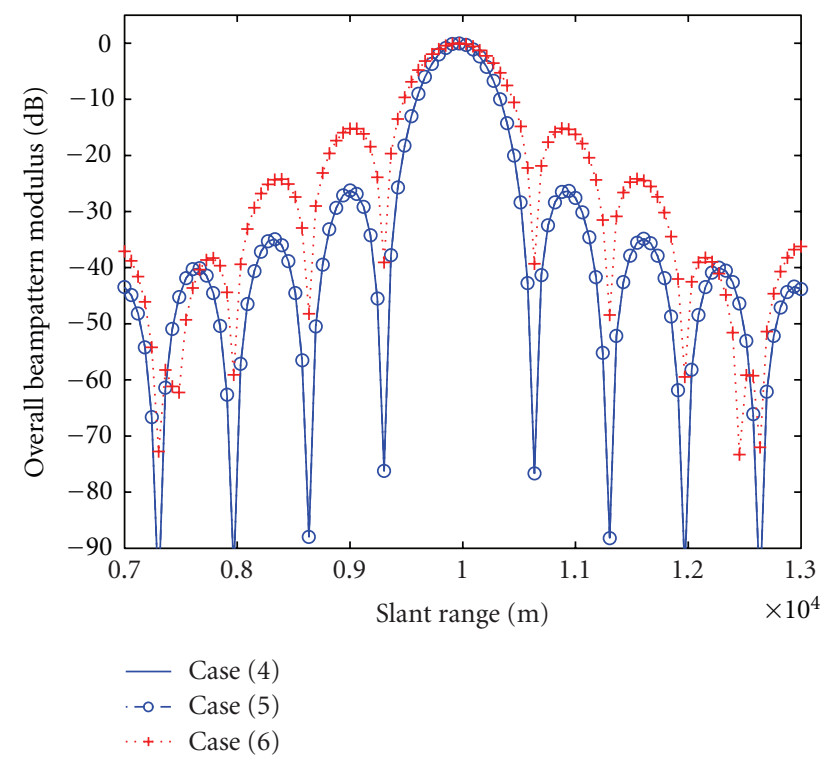

FIGURE 6: The comparative overall beampatterns in range dimension when $\Delta f \neq 0$.

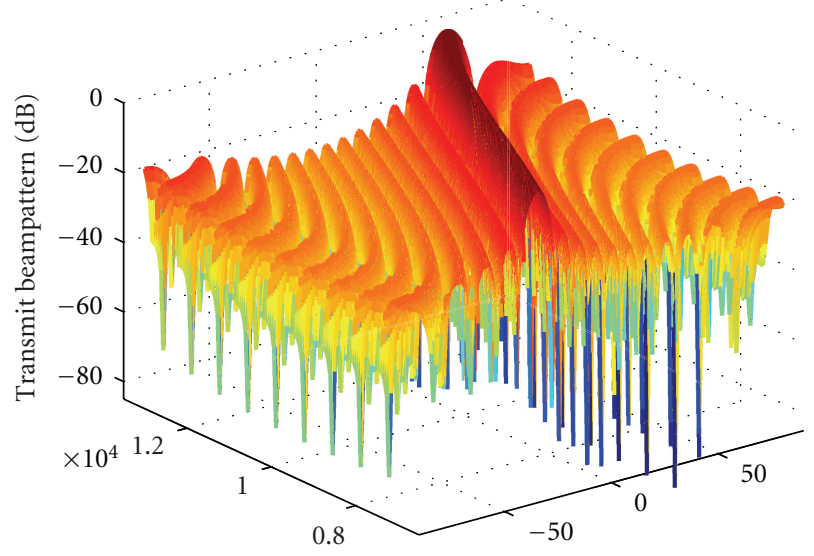

(a) $\Delta f=30 \mathrm{kHz}$

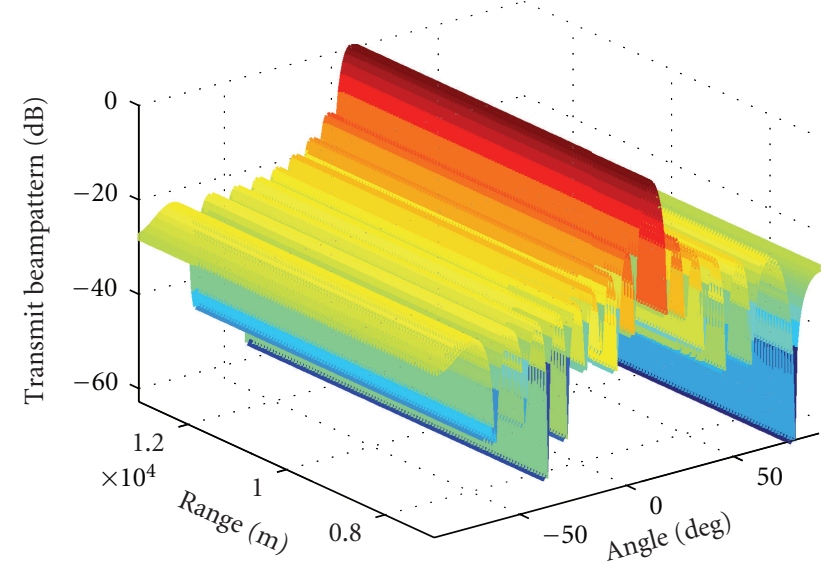

(b) $\Delta f=0 \mathrm{~Hz}$

Figure 7: The comparative transmit beampattern when $L=0.5 \lambda$.

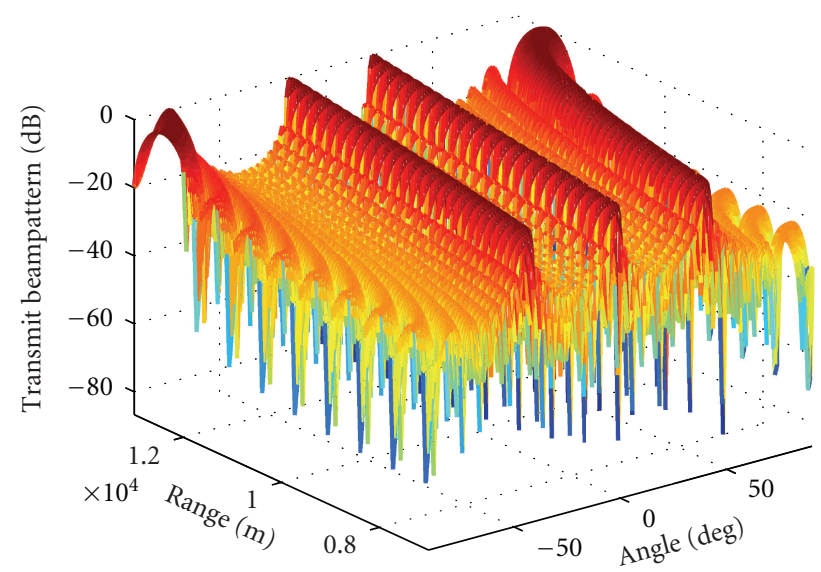

(a) $\Delta f=30 \mathrm{kHz}$

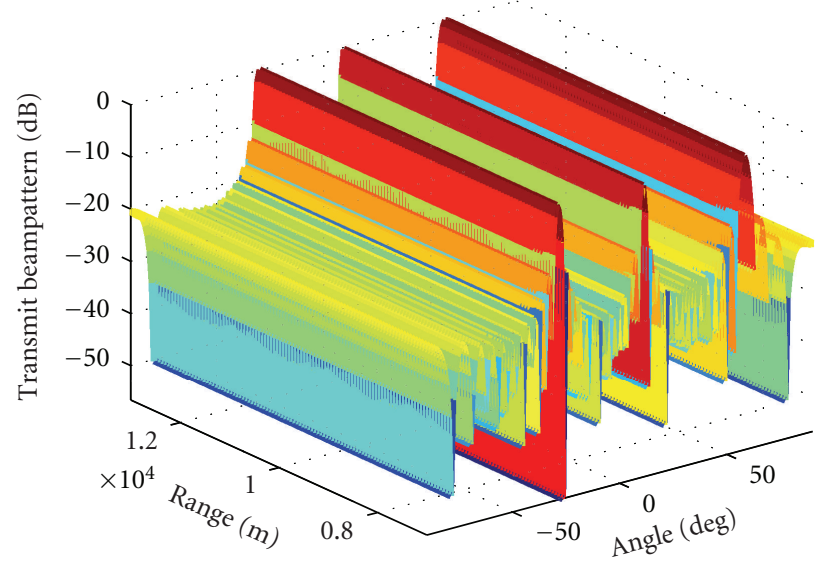

(b) $\Delta f=0 \mathrm{~Hz}$

FIGURE 8: The comparative transmit beampattern when $L=1.5 \lambda$.

phased-array which offers coherent directional gain. Besides combining the advantages of phased-array in directional gain and MIMO array in spatial diversity gain, this system also offers range-dependent beampatterns which provides many new application potentials. The system model and transmit/receive beamforming are derived. Several practical issues related to this new array are also investigated. The system performance is examined by analyzing the transmitreceive beampatterns. The proposed approach is validated by numerical simulation results. In this paper, only nonadaptive beamforming algorithms are considered. If an adaptive beamforming algorithm is used. There is a technical challenge to obtain the effective covariance matrix because the transmit beampattern is range dependent. This topic will be further investigated in our subsequent work.

\section{Acknowledgments}

This work was supported in part by the National Natural Science Foundation of China under Grant no. 41101317, the Fundamental Research Funds for the Central Universities under Grant no. ZYGX2010J001, the First Grade of 49th Chinese. Post-Doctor Research Funds under Grant no. 
20110490143, and the Open Funds of the State Laboratory of Remote Sensing. Science, Institute of Remote Sensing Applications, Chinese Academy of Sciences under Grant no. OFSLRSS201011.

\section{References}

[1] S. J. Wijnholds and W. A. Van Cappellen, "In situ antenna performance evaluation of the LOFAR phased array radio telescope," IEEE Transactions on Antennas and Propagation, vol. 59, no. 6, pp. 1981-1989, 2011.

[2] D. S. Zrnic, G. Zhang, and R. J. Doviak, "Bias correction and Doppler measurement for polarimetric phased-array radar," IEEE Transactions on Geoscience and Remote Sensing, vol. 49, no. 2, pp. 843-853, 2010.

[3] M. Bachmann, M. Schwerdt, and B. Bräutigam, "Accurate antenna pattern modeling for phased array antennas in SAR applicationsłdemonstration on TerraSAR-X," International Journal of Antennas and Propagation, vol. 2009, Article ID 492505, 9 pages, 2009.

[4] R. F. E. Guy, "Potential benefits of dynamic beam synthesis to mobile satellite communication, using the inmarsat 4 antenna architecture as a test example," International Journal of Antennas and Propagation, vol. 2009, Article ID 249602, 5 pages, 2009.

[5] W. Q. Wang, "Near-space wide-swath radar imaging with multiaperture antenna," IEEE Antennas and Wireless Propagation Letters, vol. 8, pp. 461-464, 2009.

[6] M. Younis, S. Huber, A. Patyuchenko, F. Bordoni, and G. Krieger, "Performance comparison of reflector- and planarantenna based digital beam-forming SAR," International Journal of Antennas and Propagation, vol. 2009, Article ID 614931, 13 pages, 2009.

[7] W. Q. Wang, "Space-time coding MIMO-OFDM SAR for high-resolution remote sensing," IEEE Transactions on Remote Sensing, vol. 49, no. 8, pp. 3094-3104, 2011.

[8] P. Capece, "Active SAR antennas: design, development, and current programs," International Journal of Antennas and Propagation, vol. 2009, Article ID 796064, 11 pages, 2009.

[9] W. Q. Wang, Near-Space Remote Sensing: Potential and Challenges, Springer, New York, NY, USA, 2011.

[10] J. Li and P. Stoica, "MIMO radar with colocated antennas," IEEE Signal Processing Magazine, vol. 24, no. 5, pp. 106-114, 2007.

[11] A. M. Haimovich, R. S. Blum, and L. J. Cimini, "MIMO radar with widely separated antennas," IEEE Signal Processing Magazine, vol. 25, no. 1, pp. 116-129, 2008.

[12] J. F. Zheng, Y. Li, Z. H. Feng et al., "Impact of multual coupling and polarization of antennas on BER performances of spatial multiplexing MIMO systems," International Journal of Antennas and Propagation, vol. 2012, Article ID 795205, 12 pages, 2012.

[13] Y. Gao, S. H. Wang, O. Falade, X. D. Chen, C. Parini, and L. Cuthbert, "Mutual coupling effects on pattern diversity antennas for MIMO femtocells," International Journal of Antennas and Propagation, vol. 2010, Article ID 756848, 8 pages, 2010.

[14] X. Liu and M. E. Bialkowski, "Effect of antenna mutual coupling on MIMO channel estimation and capacity," International Journal of Antennas and Propagation, vol. 2010, Article ID 306173, 9 pages, 2010.
[15] W. Q. Wang, "Applications of MIMO technique for aerospace remote sensing," in Proceedings of the IEEE Aerospace Conference, Big Sky, Mont, USA, March 2007.

[16] T. Aittomaki and V. Koivunen, "Performance of MIMO radar with angular diversity under swerling scattering models," IEEE Journal on Selected Topics in Signal Processing, vol. 4, no. 1, pp. 101-114, 2010.

[17] W. Q. Wang, "Virtual antenna array analysis for MIMO synthetic aperture radar," International Journal of Antennas and Propagation, vol. 2012, Article ID 587276, 10 pages, 2012.

[18] J. Bergin, S. McNeil, L. Fomundam, and P. A. Zulch, "MIMO phased-array for SMTI radar," in Proceedings of the IEEE Aerospace Conference (AC '08), Big Sky, Mont, USA, March 2008.

[19] J. P. Browning, D. R. Fuhrmann, and M. Rangaswamy, "A hybrid MIMO phased-array concept for arbitrary spatial beampattern synthesis," in Proceedings of the IEEE 13th Digital Signal Processing Workshop and 5th IEEE Signal Processing Education Workshop (DSP/SPE '09), pp. 446-450, Marco Island, Fla, USA, January 2009.

[20] D. R. Fuhrmann, J. P. Browning, and M. Rangaswamy, "Signaling strategies for the hybrid MIMO phased-array radar," IEEE Journal on Selected Topics in Signal Processing, vol. 4, no. 1, pp. 66-78, 2010.

[21] A. Hassanien and S. A. Vorobyov, "Phased-mimo radar: a tradeoff between phased-array and mimo radars," IEEE Transactions on Signal Processing, vol. 58, no. 6, pp. 3137-3151, 2010.

[22] M. G. Bray, D. H. Werner, D. W. Boeringer, and D. W. Machuga, "Optimization of thinned aperiodic linear phased arrays using genetic algorithms to reduce grating lobes during scanning," IEEE Transactions on Antennas and Propagation, vol. 50, no. 12, pp. 1732-1742, 2002.

[23] R. Boyer, "Performance bounds and angular resolution limit for the moving colocated MIMO radar," IEEE Transactions on Signal Processing, vol. 59, no. 4, pp. 1539-1552, 2011.

[24] J. Li, P. Stoica, L. Xu, and W. Roberts, "On parameter identifiability of MIMO radar," IEEE Signal Processing Letters, vol. 14, no. 12, pp. 968-971, 2007.

[25] R. J. Mailloux, Phased Array Antenna Handbook, Artech House, Norwood, Mass, USA, 1994.

[26] X. Zhu and R. D. Murch, "Performance analysis of maximum likelihood detection in a MIMO antenna system," IEEE Transactions on Communications, vol. 50, no. 2, pp. 187-191, 2002.

[27] W. Q. Wang, "Waveform diversity design for MIMO highresolution remote sensing," in Proceedings of the International Computational Problem-Solving Conference, Lijiang, China, December 2010.

[28] A. Hassanien and S. A. Vorobyov, "Transmit/receive beamforming for MIMO radar with colocated antennas," in Proceedings of the IEEE International Conference on Acoustics, Speech, and Signal Processing (ICASSP '09), pp. 2089-2092, Taipei, Taiwan, April 2009.

[29] B. W. Jung, R. S. Adve, J. Chun, and M. C. Wicks, "Detection performance using frequency diversity with distributed sensors," IEEE Transactions on Aerospace and Electronic Systems, vol. 47, no. 3, pp. 1800-1813, 2011.

[30] K. V. Shanbhag, D. Deb, and M. Kulkarni, "MIMO radar with spatial-frequency diversity for improved detection performance," in Proceedings of the IEEE International Communication, Control and Computing Technologies Conference, pp. 66-70, Nagercoil, India, October 2010. 
[31] J. J. Zhang and A. Papandreou-Suppappola, "MIMO radar with frequency diversity," in Proceedings of the International Waveform Diversity and Design Conference (WDD '09), pp. 208-212, Kissimmee, Fla, USA, February 2009.

[32] X.-R. Li, Z. Zhang, W.-X. Mao, X.-M. Wang, J. Lu, and W.-S. Wang, "A study of frequency diversity MIMO radar beamforming," in Proceedings of the International Conference on Signal Processing Proceedings (ICSP '10), pp. 352-356, Beijing, China, 2010.

[33] P. Antonik, M. C. Wicks, H. D. Griffiths, and C. J. Baker, "Frequency diverse array radars," in Proceedings of the IEEE Radar Conference, pp. 215-217, Verona, NY, USA, April 2006.

[34] P. Antonik, M. C. Wicks, H. D. Griffiths, and C. J. Baker, "Range dependent beamforming using element level waveform diversity," in Proceedings of the International Waveform Diversity and Design Conference, Las Vegas, Nev, USA, January 2006.

[35] P. Antonik, M. C. Wicks, H. D. Griffiths, and C. J. Baker, "Multi-mission multi-mode waveform diversity," in Proceedings of the IEEE National Radar Conference, pp. 580-582, Verona, NY, USA, April 2006.

[36] M. Secmen, S. Demir, A. Hizal, and T. Eker, "Frequency diverse array antenna with periodic time modulated pattern in range and angle," in IEEE 2007 Radar Conference, pp. 427-430, Boston, Mass, USA, April 2007.

[37] P. Baizert, T. B. Hale, M. A. Temple, and M. C. Wicks, "Forward-looking radar GMTI benefits using a linear frequency diverse array," Electronics Letters, vol. 42, no. 22, pp. 13111312, 2006.

[38] X. Liu and L. Zhuang, "Application of frequency diversity to suppress grating lobes in coherent MIMO radar with separated subapertures," Eurasip Journal on Advances in Signal Processing, vol. 2009, Article ID 481792, 2009.

[39] B. D. Steinberg and E. H. Attia, "Sidelobe reduction of random arrays by element position and frequency diversity," IEEE Transactions on Antennas and Propagation, vol. 31, no. 6, pp. 922-930, 1983.

[40] B. D. Steinberg, "The peak sidelobe of the phased array having ramdomly located elements," IEEE Transactions on Antennas and Propagation, vol. 20, no. 2, pp. 129-137, 1972.

[41] F. E. Nathanson, J. P. Reilly, and M. N. Cohen, Radar Design Principles: Signal Processing and the Environment, McGrawHill, New York, NY, USA, 1990.

[42] W. Q. Wang, Q. Peng, and J. Cai, "Waveform-diversity-based millimeter-wave UAV SAR remote sensing," IEEE Transactions on Geoscience and Remote Sensing, vol. 47, no. 3, pp. 691-700, 2009.

[43] H. L. Van Trees, Optimum Array Processing, John Wiley \& Sons, New York, NY, USA, 2002.

[44] J. Farooq, M. A. Temple, and M. A. Saville, "Application of frequency diverse arrays to synthetic aperture radar imaging," in 2007 International Conference on Electromagnetics in Advanced Applications, ICEAA'07, pp. 447-449, Turin, Italy, September 2007.

[45] J. Farooq, M. A. Temple, and M. A. Saville, "Exploiting frequency diverse array processing to improve SAR image resolution," in 2008 IEEE Radar Conference (RADAR '08), Rome, Italy, May 2008. 

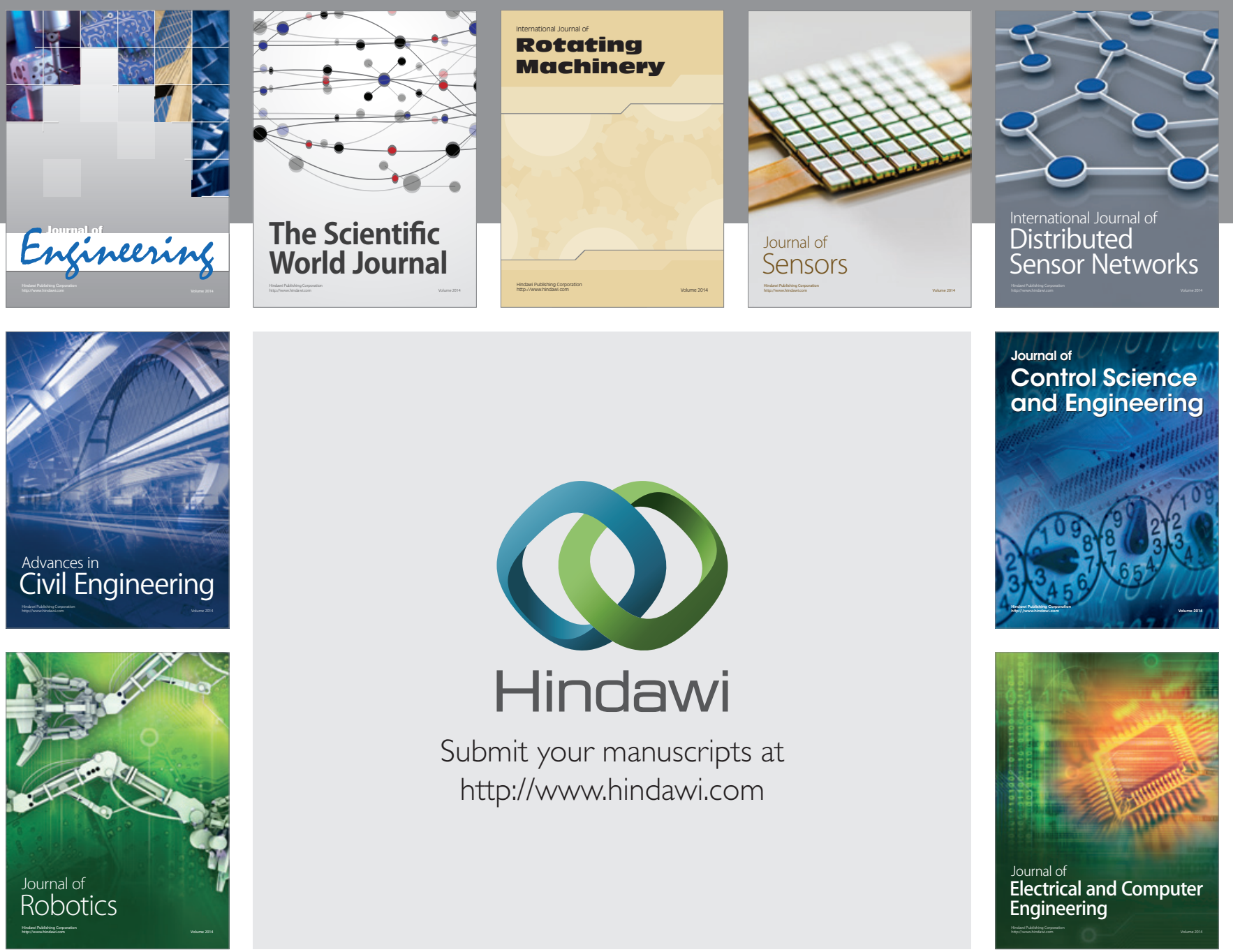

Submit your manuscripts at

http://www.hindawi.com
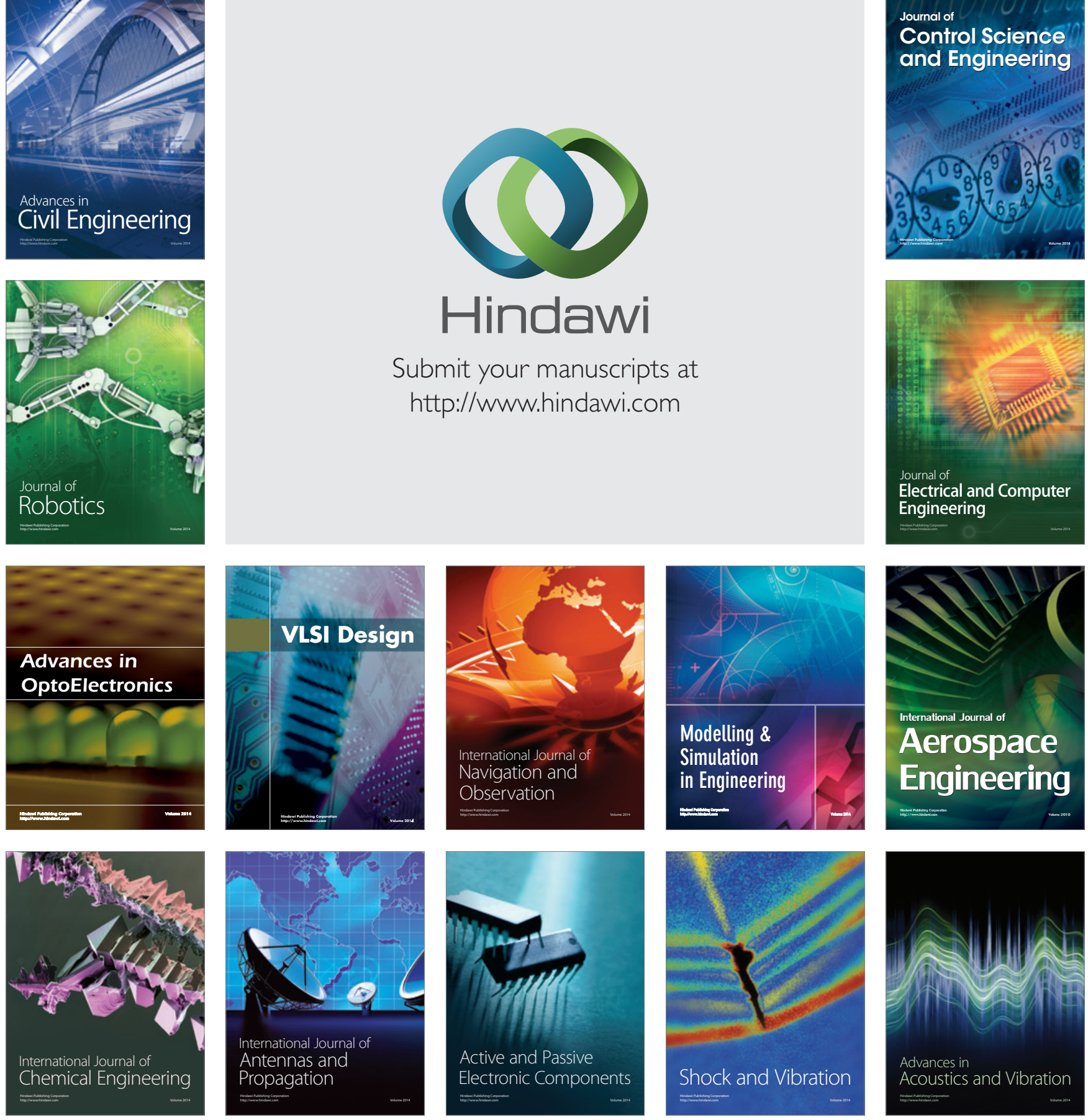